\title{
Medium-Energy Ion Scattering study of strained holmium silicide nanoislands grown on silicon (100)
}

\author{
T.J. Wood, ${ }^{1}$ C. Eames, ${ }^{1}$ C. Bonet,${ }^{1}$ M. B. Reakes, ${ }^{1}$ T.C.Q. Noakes,${ }^{2}$ P. Bailey, ${ }^{2}$ and S.P. Tear ${ }^{1, *}$ \\ ${ }^{1}$ Department of Physics, University of York, Heslington, York, YO10 5DD, UK \\ ${ }^{2} S T F C$ Daresbury Laboratory, Daresbury, Warrington, WA4 4AD, UK
}

(Dated: November 7, 2018)

\begin{abstract}
We have used medium-energy ion scattering (MEIS) to quantitatively analyse the structure of holmium silicide islands grown on the $\mathrm{Si}(100)$ surface. Structure fitting to the experimental data unambiguously shows that the tetragonal silicide phase is present and not the hexagonal phase which is associated with the growth of nanowires at submonolayer coverages. Islands formed with a lower holmium coverage of $3 \mathrm{ML}$ are also shown to be tetragonal which suggests that the hexagonal structure is not a low coverage precursor to the growth of the tetragonal phase. MEIS simulations of large nanoislands which include the effects of lateral strain relief have been performed and these compare well with the experimental data.
\end{abstract}

Whilst there have been many studies of rare-earth (RE) silicides on the $\mathrm{Si}(111)$ surface, relatively little was known about the growth mechanisms on the $\mathrm{Si}(100)$ surface until the discovery of self-assembled nanowires by Preinesberger et al. 1] These novel structures form when a suitable RE metal, eg. Gd, 2, 3, 4, 5, 6] Sc, [4] Dy, [1, 7, 8, 9, 10] Sm, 4] Er, 7, 8, 11, 12, 13] Ho [8, 14] or $\mathrm{Y}, 15$ is deposited onto a clean $\mathrm{Si}(100)$ substrate held at an elevated temperature. Characteristic 'wires' measuring up to a micrometre in length, and typically only a few nanometres wide were observed by scanning tunneling microscopy (STM). This discovery and the potential technological applications of such conducting nanowires has motivated considerable interest over the last ten years 2., 3, 4, 5, 6, 7, 8, 9, 10, 11, 12, 13, 14, 15, 16, 17, 18, 19, 20, 21, 22, 23, 24, 25, 26, 27, 28]. Chen et al. have demonstrated that the growth of nanowires with extremely high aspect ratios is the result of the anisotropic lattice mismatch that results from the growth of the hexagonal, defect- $\mathrm{AlB}_{2} \mathrm{RE}$ silicide on the $\mathrm{Si}(100)$ surface (see Fig. (1) [11].

Depositing more RE metal causes islands to form with the surface displaying a $\mathrm{c}(2 \times 2)$ periodicity. The structure of these $\mathrm{RE}$ silicide islands has been proposed to be either hexagonal, tetragonal $\left(\mathrm{ThSi}_{2}\right)$ or orthorhombic $\left(\mathrm{GdSi}_{2}\right)$ 11, 6, 10, 12, 13, 16, 17, 22, 27, 28]. All three of these phases are known to exist in the bulk and their lattice constants have been measured and it is known that the bulk orthorhombic phase is only a small distortion of the tetragonal phase [29]. A side-view schematic of the $\mathrm{ThSi}_{2}$ structure is shown in Fig. 2. Evidence for the growth of the tetragonal form has generally been inferred from lattice mismatch arguments, along with STM measurements of step heights in the silicides on the $\mathrm{Si}(100)$ surface [13]. SXRD measurements also yield information which appears to confirm that Er silicide islands adopt the tetragonal form, with the surface $\mathrm{c}(2 \times 2)$ periodicity

*e-mail: spt1@york.ac.uk

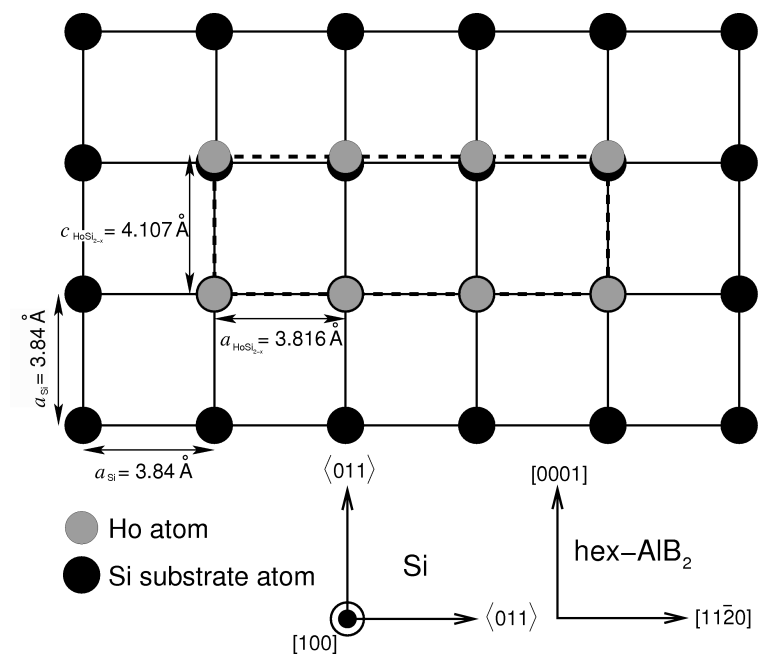

(a)

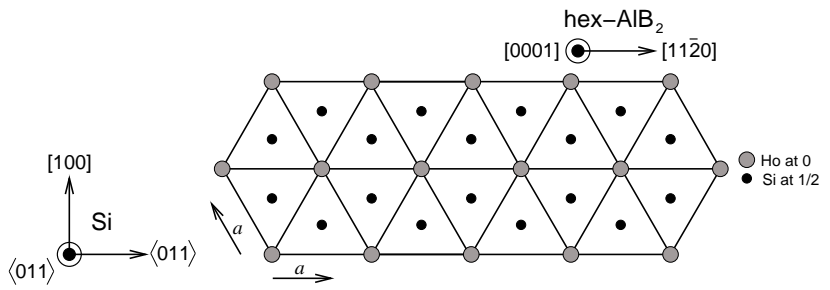

(b)

FIG. 1: (a) A top down schematic illustrating the lattice matching of the hexagonal defect- $\mathrm{AlB}_{2}$ structure to the $\mathrm{Si}(100)$ surface. The anisotropy in lattice mismatch creates the high aspect ratio nanowires commonly observed for several RE metals. (b) A side view schematic of the $\mathrm{AlB}_{2}$ structure.

interpreted as being due to Si adatoms [12].

STM experiments reveal that the island morphology is very sensitive to the $\mathrm{RE}$ metal deposited and to the annealing temperature used. Those RE metals (Nd, Sm, $\mathrm{Yb}$ ) that have a low anisotropy in their lattice match to the substrate form compact 3D islands [8]. Those that have a high anisotropy form both elongated and compact islands, depending upon the growth temperature. 


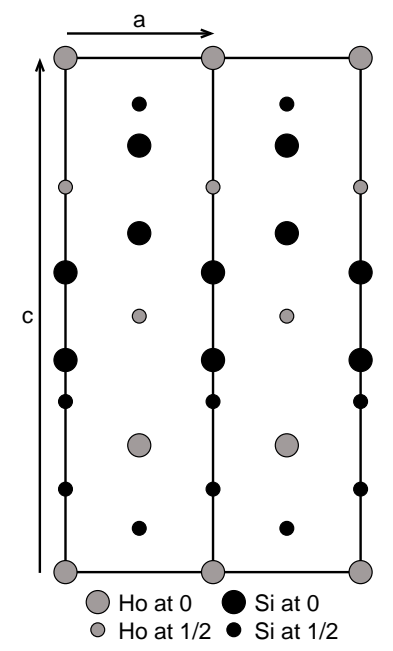

FIG. 2: A side view schematic of the tetragonal $\mathrm{ThSi}_{2}$ structure. The orthorhombic $\mathrm{GdSi}_{2}$ structure of $\mathrm{HoSi}_{2}$ is essentially the same but with $a=4.03 \AA$ and $b=3.94 \AA$, a difference of just $2.25 \%$.

Growth at lower temperatures $\left(600^{\circ} \mathrm{C}\right)$ causes the formation of elongated islands and growth at temperatures above $650^{\circ} \mathrm{C}$ results in compact $3 \mathrm{D}$ islands. However, the two island morphoplogies have been observed to coexist, especially when using intermediate annealing conditions.

Dysprosium silicide provides an interesting case study. The elongated islands have typical dimensions $2 \mathrm{~nm}$ high, $15 \mathrm{~nm}$ wide, and $500 \mathrm{~nm}$ long and the smaller compact islands have typical dimensions $5 \mathrm{~nm}$ high, $50 \mathrm{~nm}$ wide and 200nm long 22]. Using high resolution cross-sectional TEM, Ye et al. demonstrated that elongated and nonelongated islands coexist when Dy silicide is grown at 600 ${ }^{\circ} \mathrm{C}-650^{\circ} \mathrm{C}$ on the $\mathrm{Si}(100)$ surface 27 . They proposed that the structure of the elongated islands was hexagonal, and the excessive stress within this structure (due to the large $c$-axis mismatch) is relieved through dislocations and tilting across the width of the island. The non-elongated islands were found to be a tetragonal or orthorhombic structure with only a small amount of tilting required to relieve the stress, since the lattice match is relatively small in both directions. It was also noted that there was some expansion of the $c$-axis to relieve the stress in much the same way as has been demonstrated for the 2D and 3D RE silicides on $\operatorname{Si}(111)$ 30, 31]. However, the recent HR-TEM study by He et al. claims that the compact 3D Dy silicide islands are in fact a fully relaxed, and hence stress free, hexagonal form, whilst the elongated nanowire islands are tetragonal/orthorhombic, with the faulted stacking relieving the stress in the structure 22. The delicate energy balance within the silicide in this particular example is also highlighted by the fact that there is both a tetragonal and an orthorhombic phase of Dy silicide in the bulk.

Despite the many studies that have been conducted,

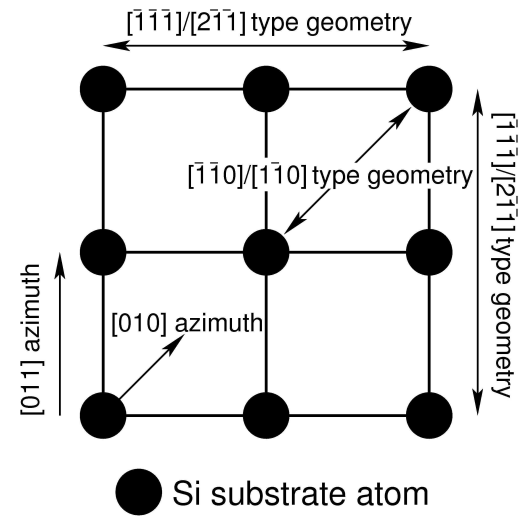

FIG. 3: View from above of the bulk terminated Si(100) surface showing the two incident beam directions used in the MEIS experiments. For the $[\overline{1} \overline{1} 0] /[1 \overline{1} 0]$ geometry the angle with respect to the sample normal was $45^{\circ}$ and for the $[\overline{1} \overline{1}] /[2 \overline{1} \overline{1}]$ geometry the polar angle was $54.74^{\circ}$. The scattering plane through the tetragonal silicide is shown for the $[\overline{1} \overline{1} 0] /[1 \overline{1} 0]$ geometry in figure 9 .

it has been noted in recent work that no ideal crystallographic determination has been possible to clearly prove once and for all the true nature of the 3D islands 22, 26, 28] created when the rare earth metals form a silicide in this way. MEIS is of particular value in the study of these nanostructures since the large mass contrast of Ho and Si allows the elucidation of the silicide structure free from substrate effects. This also means that the technique is able to isolate the regions of interest on the surface by only selecting the Ho signal for the structural optimisation.

\section{EXPERIMENT}

All MEIS data were obtained at the UK MEIS facility at STFC Daresbury Laboratory. The experiments were conducted under ultra-high vacuum (UHV), with typical base pressures of $2 \times 10^{-10}$ mbar. Clean $\mathrm{Si}(100)$ samples (cut from a lightly doped n-type wafer) were prepared by $e$-beam heating to $\sim 1200{ }^{\circ} \mathrm{C}$ for $1 \mathrm{~min}$, then slowly cooled $\left(<100{ }^{\circ} \mathrm{C} \mathrm{min}{ }^{-1}\right)$ between $1000{ }^{\circ} \mathrm{C}$ and $600{ }^{\circ} \mathrm{C}$ to ensure an ordered surface was obtained. Temperature measurements were made using an infrared pyrometer. The heating cycles were repeated until a sharp $2 \times 1$ lowenergy electron diffraction (LEED) pattern was observed. The 3D silicide islands were then formed by depositing approximately 6 ML of Ho from a quartz crystal calibrated tantalum boat source onto this clean $\mathrm{Si}(100)$ surface, which was held at $\sim 650{ }^{\circ} \mathrm{C}$ during deposition and for 5 min afterwards. When a c $(2 \times 2)$ LEED pattern was observed and the Auger electron spectrum showed the samples to be free from contamination, they were transferred under UHV into the ion scattering chamber.

The ion scattering data were taken using $100 \mathrm{keV} \mathrm{H}^{+}$ 
ions incident upon the sample and the scattered ions were detected using an angle-resolving toroidal-sector electrostatic ion-energy analyser and its microchannel plate detector. The MEIS spectra confirmed that the samples were free of contaminants and data were acquired with a total dose of $\sim 10^{16}$ ions $\mathrm{cm}^{-2}$. Further details about the Daresbury MEIS facility can be found in the literature [30, 32, 33, 34, 35]. During each experiment two different incident beam directions onto the sample were used. These beam directions are shown with respect to the substrate in fig. 3. The notation $[$ in $] /[$ out $]$ for each geometry defines the ingoing crystal direction $[\mathrm{in}]$ and an outgoing crystal direction [out] that lies in the detected angular range of the scattered ions.

\section{STRUCTURE FITTING: TETRAGONAL OR HEXAGONAL?}

Due to the contention in the literature regarding whether these islands take the hexagonal, tetragonal or orthorhombic form, MEIS proves particularly powerful since it can clearly demonstrate the presence of one of these structures. In this work we have used the XVegas code [36] which uses Monte-Carlo methods to simulate the blocking curves of a proposed structural model.

Fig. 4(a) shows the simulated blocking curves obtained when differing numbers of layers of $\mathrm{RE}$ are present in the hexagonal structure for the $[\overline{1} \overline{1} 0] /[1 \overline{1} 0]$ geometry. The interlayer separation of all the Ho layers has been optimised to give the best visual agreement with experimental data, with the two intermediate Si layers lying $1 / 3$ and $2 / 3$ of this layer separation from the Ho layer beneath. A HoHo layer separation of $3.28 \AA$ has been found to give the best-fit. However, the quality of the fits obtained is very poor, clearly demonstrating that this is not the correct structure. To make this clearer, the fit for 6 layers of Ho is shown in Fig. 4(b) At scattering angles of $88^{\circ}, 90^{\circ}$, $100^{\circ}, 106^{\circ}, 118^{\circ}, 127$ and $142^{\circ}$ there are major discrepancies between the experimental data and the best-fit simulations. The fully relaxed hexagonal structure that was proposed by He et al. 222] does not explain the experimental blocking curves either, as such a structure would produce almost identical blocking curves, but would yield a smaller $c$-axis of the silicide.

The tetragonal structure has also been fitted to the data, since it is assumed that the silicide takes the same $a$ and $b$ values as the Si substrate to form an epitaxial overlayer. This goes against the available data in the literature regarding the structure of the bulk Ho silicides, as no tetragonal phase has been observed (though the orthorhombic $\mathrm{GdSi}_{2}$ form has been reported with lattice parameters of $a=4.03 \AA, b=3.944 \AA$ and $c=13.30 \AA$ ) [29]. Conducting simulations of such a tetragonal structure gives the fits for the $[\overline{1} \overline{1} 0] /[1 \overline{1} 0]$ geometry shown in Fig. 5(a) which are for structures that are one, two and three $\mathrm{ThSi}_{2}$-type cells in height. It is clear that a crystal with a depth of two $\mathrm{ThSi}_{2}$ cells produces the best

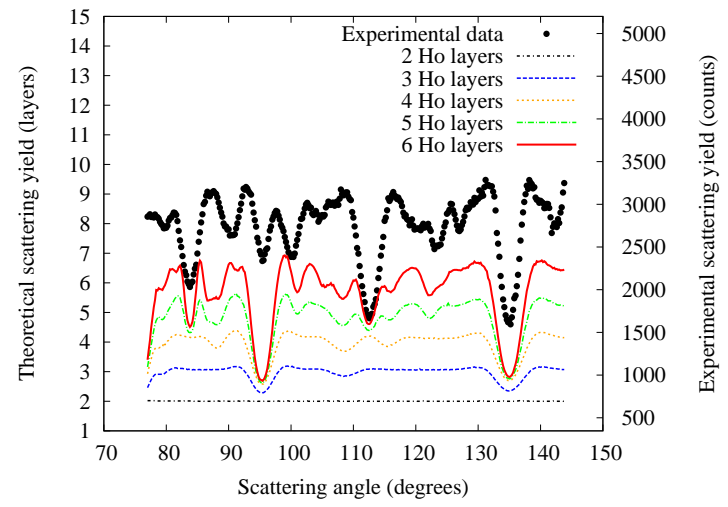

(a)

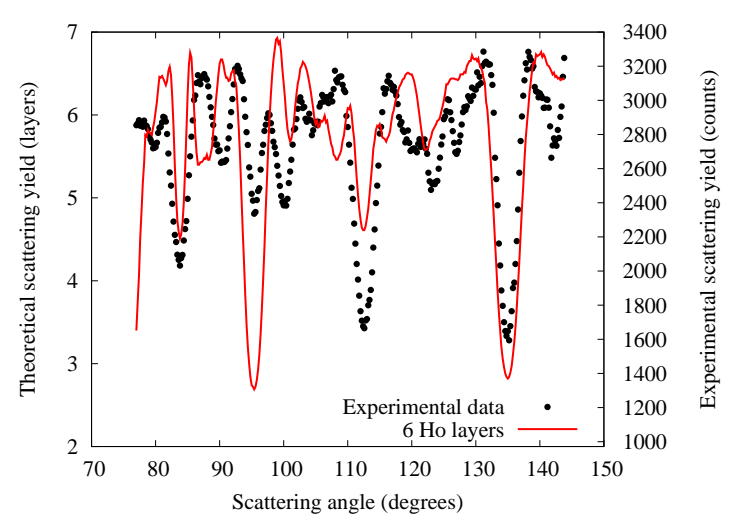

(b)

FIG. 4: Original in colour. (a) A comparison of the simulations of the hexagonal structure with 2-6 layers of Ho, to the experimental data for the $[\overline{1} \overline{1} 0] /[1 \overline{1} 0]$ geometry. (b) The fit obtained for 6 Ho layers in the hexagonal structure, with a Ho-Ho layer separation of $3.28 \AA$.

match. The detailed structure fits are shown in figures $5(\mathrm{~b})$ and 5(c). The r.m.s. thermal vibrations of the Ho atoms were set to the bulk metal value of $0.13 \AA$, whilst a fitting of the Si vibrations gives an enhanced value of $0.15 \AA$. These vibrations are consistent with the previous MEIS studies of 2D and 3D RE silicides on $\mathrm{Si}(111)$ [31, 35], with the factor of two enhancement for the Si atoms possibly indicating the presence of static disorder in these layers. This could be due to the presence of $\mathrm{Si}$ vacancies [20, 25].

Optimising the layer separation of the Ho atoms gives the best visual agreement for both geometries when $c=$ $13.14 \AA$. It is clear from comparing fig. [5(b) with fig. 4(b) that the tetragonal form of the silicide is a better fit to the data than the hexagonal structure.

\section{AN ANOMALOUS C-AXIS VALUE}

The assumption that the silicide grows epitaxially on the $\mathrm{Si}(100)$ surface yields a constant $c$-axis value throughout this silicide $(13.14 \AA)$ that is much smaller than the 


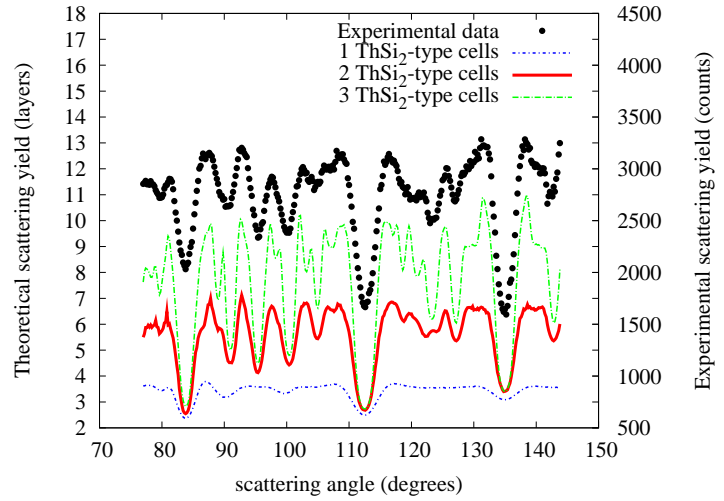

(a)

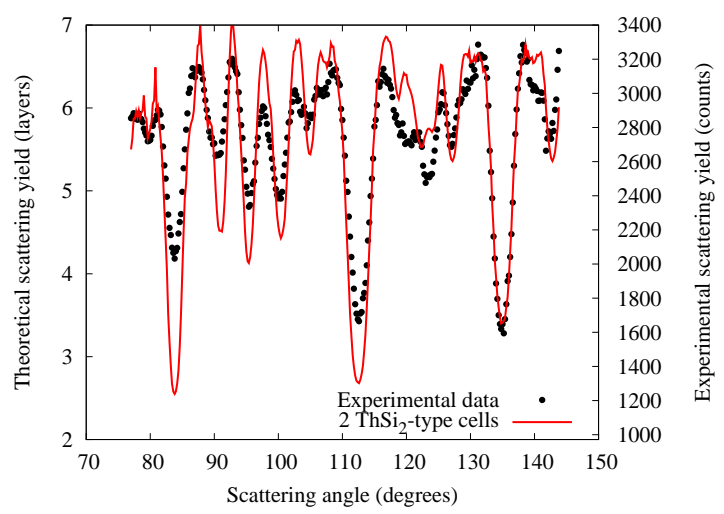

(b)

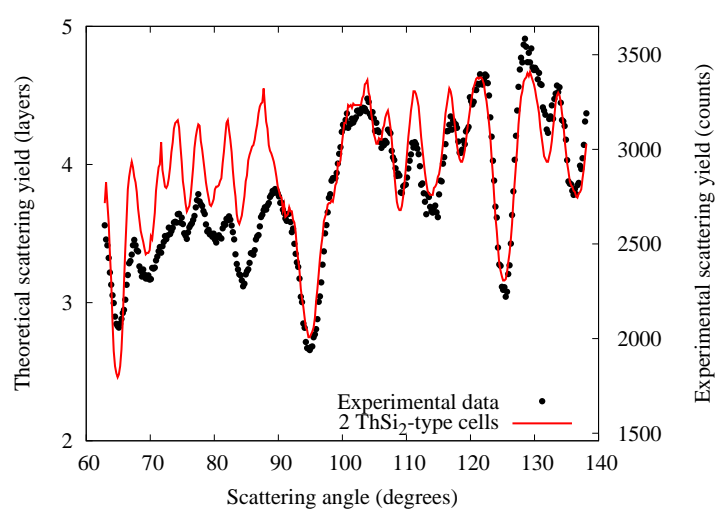

(c)

FIG. 5: Original in colour. (a) Comparison of simulations of the tetragonal structure with thicknesses of $2-4 \mathrm{ThSi}_{2}$ cells in height to the experimental data for the $[\overline{1} \overline{1} 0] /[1 \overline{1} 0]$ geometry. (b) The fit obtained for the $[\overline{1} \overline{1} 0] /[1 \overline{1} 0]$ geometry when the silicide is two $\mathrm{ThSi}_{2}$ cells in height. (c) The fit obtained for the $[\overline{1} \overline{1} \overline{1}] /[2 \overline{1} \overline{1}]$ geometry when the silicide is two $\mathrm{ThSi}_{2}$ cells in height.

value of $13.30 \AA$ for the bulk silicide. We would expect that the stress induced through a contraction of the Ho silicide $a$ - and $b$-axes at the interface, would be released through an expansion in the perpendicular direction and such behaviour has been directly observed in other rare earth silicide surfaces [30, 31]. In particular, Ye et al. have reported an expansion in the $c$-axis of the silicide relative to the bulk value for $\mathrm{Dy}$ on $\operatorname{Si}(100)$ [27].

One explanation of this observation could be that the silicide is incommensurate with the $\mathrm{Si}$ substrate. Hence it would seem reasonable to assume that the silicide takes the orthorhombic form that is observed for bulk Ho silicide. Refitting the experimental data to simulations of this structure, where $a=4.030 \AA$ and $b=3.944 \AA$, yields almost identical fits, but with a $c$-axis of $13.70 \AA$. This large value of the silicide $c$-axis does not make sense according to the established trend in strain either, as it would be expected that the lack of strain at the interface would not cause any expansion in the $c$-axis of the silicide relative to the bulk value. Thus it can be concluded that it is not the bulk form of the silicide that is present on the surface; it must be some intermediate form.

One of the limitations of MEIS when only using the Ho blocking curves to solve crystallographic structure is that it is not possible to exclusively determine both the $a$ - and $c$-axis lattice constants of the silicide. This is because any difference in the silicide $a$-axis could be compensated by a change in the $c$-axis to produce blocking curves with blocking dips in the same positions. Hence, a particular $c / a$ ratio will yield a series of blocking curves that are exactly the same, even though the actual lattice parameters may be very different. We have performed a series of simulations, each with a fixed $a$-axis, in which the $c$-axis has been optimised by varying the layer separation of a silicide that is two $\mathrm{ThSi}_{2}$ cells in height. These results are shown in Table 【.

\begin{tabular}{cccc}
$a(\AA)$ & $c_{110}( \pm 0.04 \AA)$ & $c_{111}( \pm 0.04 \AA)$ & $c_{\text {average }}( \pm 0.04 \AA)$ \\
\hline 3.84 & 13.12 & 13.16 & 13.14 \\
3.88 & 13.24 & 13.28 & 13.26 \\
3.92 & 13.40 & 13.44 & 13.42 \\
3.96 & 13.52 & 13.56 & 13.54 \\
4.00 & 13.68 & 13.72 & 13.70 \\
\hline
\end{tabular}

TABLE I: Optimised $c$-axis values determined for various fixed values of the $a$-axis. Fitting the $[\overline{1} \overline{1} 0] /[1 \overline{1} 0]$ and $[\overline{1} \overline{1} \overline{1}] /[2 \overline{1} \overline{1}]$ geometries yield slightly different values, which can be averaged to give an overall best-fit value.

The data from Table \ are plotted in Fig. 6 and the straight line fit defines a $c / a$ ratio of $3.42 \pm 0.01$. The positive gradient suggests that as the lateral lattice constant of the tetragonal unit cell is reduced to match that of the substrate, the lattice constant perpendicular to the surface also contracts and this is true for all pairs of $c$, $a$ values. As noted earlier, we might expect the opposite to occur and the $c$-axis to expand in order to maintain the volume of the unit cell. 


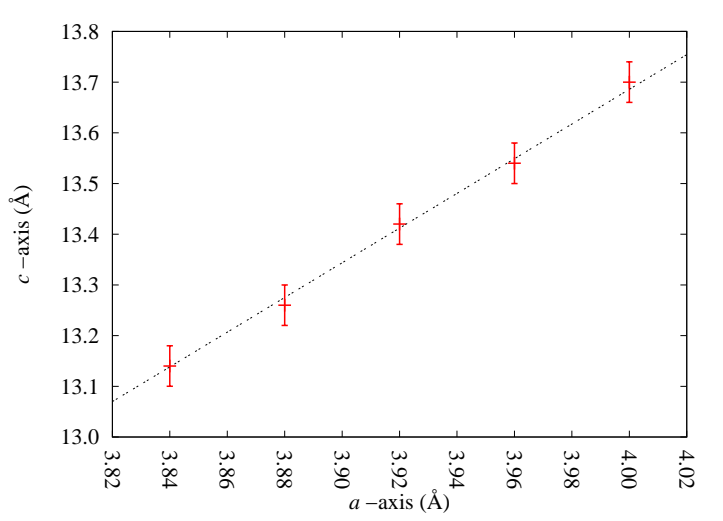

FIG. 6: A plot showing how the $c$-axis determined is dependent on the size of the $a$-axis of the silicide. Fitting a straight line which passes through the origin yields a $c / a$ ratio of 3.42 \pm 0.01 .

\section{SIMULATION OF STRAINED ISLANDS}

There is another reason that might explain the unusually low $c$-axis suggested by the fitting procedure. If the lateral lattice constant of the unit cell is constrained to match that of the substrate at the interface and is allowed to expand to relieve strain as we move away from the interface then the blocking angles will be shifted. The average $a$-axis throughout the structure will increase and so will the fitted $c$-axis in order to maintain the $c / a$ ratio of 3.42. This effect would allow for interfacial matching and would at the same time provide a more physically meaningful thickness for the silicide.

Simulation of this effect is computationally intensive. Unit cells with lateral strain as a function of height do not laterally tesselate ad-infinitum and a structure with finite lateral extent must be considered. This must be large enough to approach the size of a typical surface island but small enough to be computationally tractable. We have taken a unit cell with a $10 \times 10$ lateral extent, making an island $38.4 \times 38.4 \AA^{2}$. This is two tetragonal unit cells deep and in total includes 2500 atoms. Computational resources prohibit us from a full optimisation of the strained structure in terms of interlayer spacings or vibrational amplitudes. Instead we have taken values suggested from the structure fitting carried out on the $1 \times 1 \times 2$ cells detailed earlier in this work. The lateral lattice constants are allowed to relax from a value of $3.84 \AA$ at the bottom of the supercell (interfacial matching to $\operatorname{Si}(100)$ ) to $3.99 \AA$ at the top of the supercell (the averaged unstrained lateral lattice constant of bulk orthorhombic holmium silicide). The strain relief is just a linear function of height and, for example, we have not considered a case in which only the few layers near the interface are significantly strained. The strained island is shown from above in Fig. 7

Since we cannot currently fully optimise the $c$-axis in such a strained island we have simulated the blocking

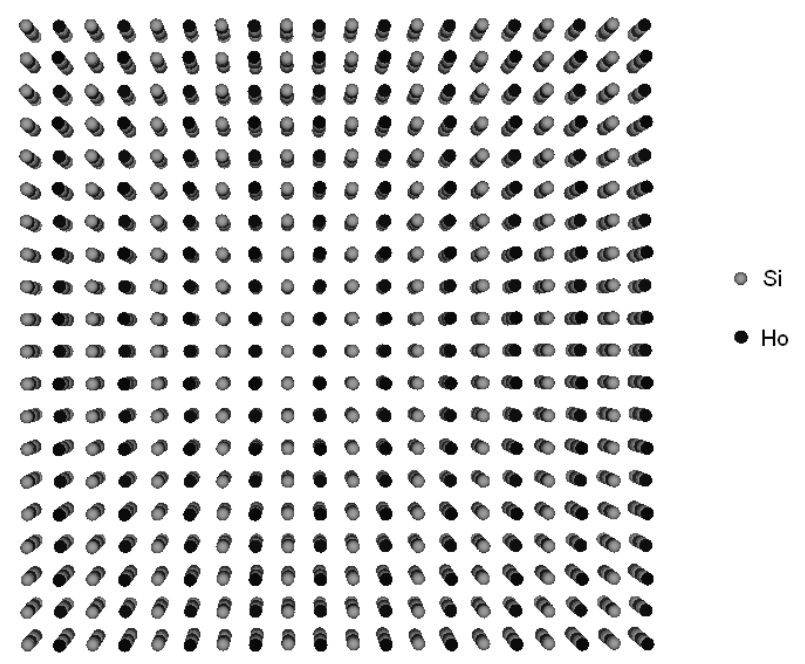

FIG. 7: View from above of the $10 \times 10 \times 2$ unit cell showing the lateral strain.

curves for four such islands, each with a different depth. This depth is varied by choosing two different $c$-axes for the two unit cells that form the island. These four $c$-axis values are; 1 ) both as bulk $c$-axis values (13.30 $\AA$ ), 2) both at the values obtained earlier in structure fitting with $1 \times 1 \times 2$ cells with no strain relief $(13.14 \AA), 3)$ expansion of the $c$-axis of the cell nearest the interface by $2 \%$ and a bulk $c$-axis value in the top unit cell (13.57 $\AA, 13.30$ $\AA$ ) and 4) expansion of the $c$-axis of the cell nearest the interface by $4 \%$ and the top cell by $2 \%$ (13.83 $\AA, 13.57 \AA)$. The calculated blocking curves are shown in Fig. 8 for the $[\overline{1} \overline{1} 0] /[1 \overline{1} 0]$ geometry, the $[\overline{1} \overline{1} \overline{1}] /[2 \overline{1} \overline{1}]$ geometry not being calculated because it would require averaging over two possible domains and thus twice as many simulations.

On first viewing the incorporation of strain appears to make the fit worse (compare with the fit for the $1 \times 1 \times 2$ cell in Fig. 5(b)]. The region with a scattering angle in the approximate range $117^{\circ}-129^{\circ}$ is especially bad. The scattering plane is shown schematically in Fig. 9. We can see from this that in the regions where the fit is bad it is the positions of the silicon atoms that are responsible for the poor fit of the blocking dips. Another minor silicon atom blocking dip at around $107^{\circ}$ is also a poor fit. Given that the positions and the thermal vibrations of the silicon atoms have not been optimised it is no surprise that the fit is made worse by the introduction of strain.

However, the blocking dips produced by this structure are dominated by those caused by holmium atoms and close inspection of the major blocking dips at roughly $82^{\circ}, 113^{\circ}$ and $135^{\circ}$ supports the possibility of strain in these structures. The detail around these blocking dips is shown in Fig. 10. In the strained cells as the $c$-axes are changed from the unrealistically low value obtained in the fit on an unstrained cell (13.14 $\AA$ ) through the bulk silicide value $(13.30 \AA)$ and into the two cases where the 


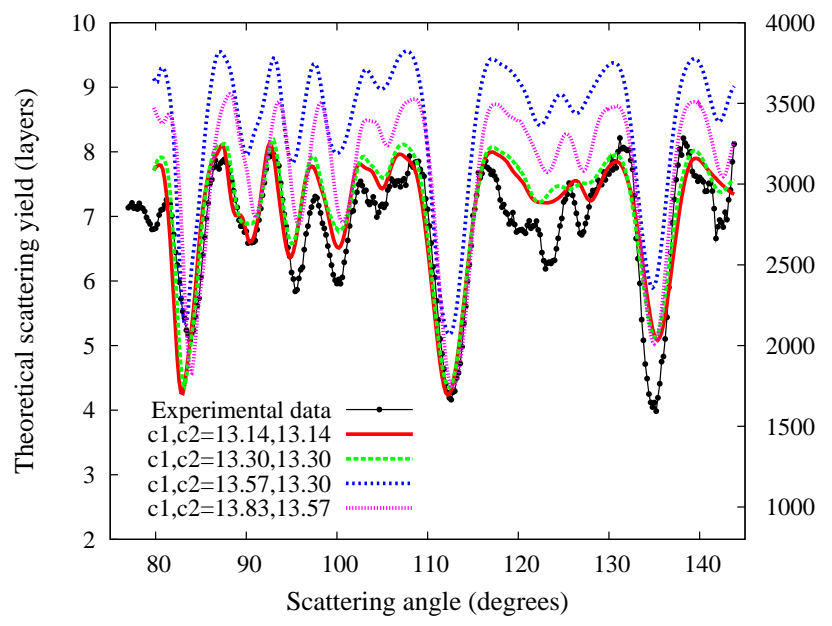

FIG. 8: Original in colour. Comparison of simulated strain in large $10 \times 10 \times 2$ unit cells with experiment. The lateral lattice constant of each unit cell that makes up the nanoisland was allowed to relax from $3.84 \AA$ (interface lattice matching with $\mathrm{Si}(100))$ to $3.99 \AA$ on the surface (bulk lattice constant). The four simulations plotted here correspond to fixed values of the two $c$-axes in the $10 \times 10 \times 2$ unit cell. The first unit cell (c1) is nearest the surface and the second (c2) is nearest the interface.

$c$-axis is expanded the blocking dip is shifted to become closer and closer to the experimentally measured dip. Indeed, at $84^{\circ}$ and $113^{\circ}$ the blocking dip for the strained cells is a better fit to experiment than that for the unstrained, fully optimised and multilayer averaged fit.

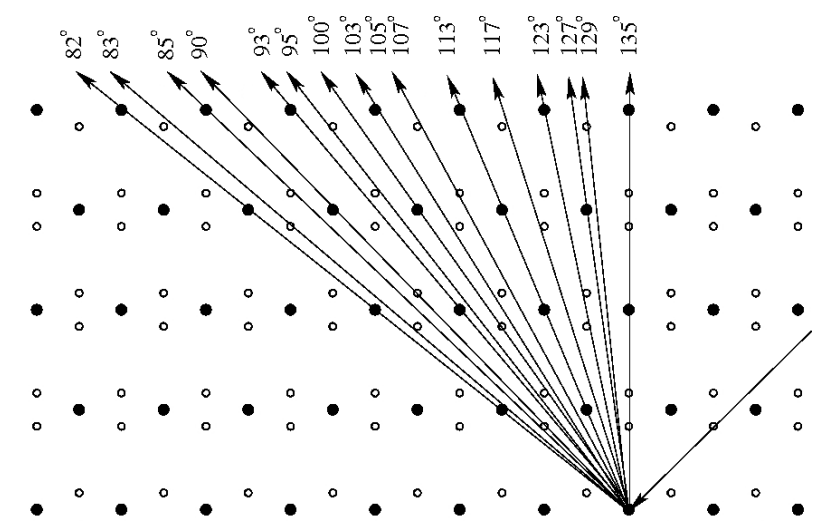

FIG. 9: Schematic slice showing the scattering plane for the $[\overline{1} \overline{1} 0] /[1 \overline{1} 0]$ geometry and the atoms responsible for the principal blocking dips observed. Filled circles represent Ho atoms and empty circles silicon atoms.

Thus far, the question of the surface termination has not been addressed. The unit cells involved in the simulations are holmium terminated which is not physically reasonable. Also, the LEED pattern shows a $\mathrm{c}(2 \times 2)$ periodicity which is not accounted for in any of the simulated cells. We have attempted to investigate the surface

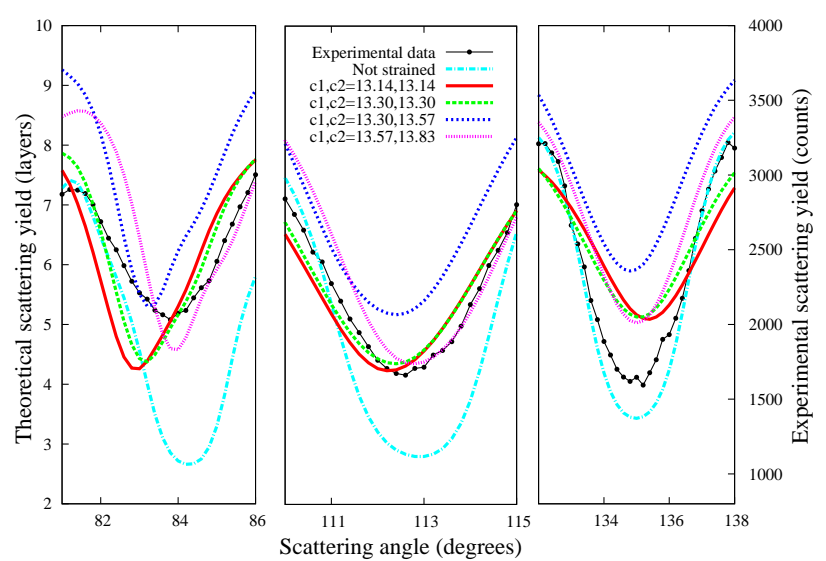

FIG. 10: Original in colour. Showing the detail around the major blocking dips in Fig. 8 With the gradual introduction of larger, more physically reasonable $c$-axis values into the strained cells the fit is improved until it is better than that for the fully optimised but non-strained cell.

termination but the simulated blocking curves show very little sensitivity to an extra layer of silicon atoms that form a surface termination. Other experiments that are more sensitive to the very top atomic layer may be better suited to resolving the issue of the surface termination (such as MEIS itself at normal incidence with $50 \mathrm{keV} \mathrm{He}$ ions).

\section{LOWER COVERAGES}

The work presented here suggests that at a coverage of $6 \mathrm{ML}$ and under our preparation conditions strained tetragonal silicide islands are formed when holmium is grown on $\mathrm{Si}(100)$. At lower coverages $(<1 \mathrm{ML})$ nanowires form that are believed to be related to the hexagonal structure. The question naturally arises as to what structure forms in the intermediate coverage regime. Does the tetragonal structure grow atop the hexagonal phase? Or is there a phase change at a certain minimum coverage which results in tetragonal rather than hexagonal growth? To attempt to answer these question we have taken experimental data at a coverage of $3 \mathrm{ML}$ under the same growth conditions. Fig. 11 shows these data from the $3 \mathrm{ML}$ sample compared with that from the sample grown using $6 \mathrm{ML}$ of holmium.

The principal blocking dips at $113^{\circ}$ and $135^{\circ}$ are present in both samples. In the $6 \mathrm{ML}$ sample there are blocking dips at $90^{\circ}, 95^{\circ}$ and $100^{\circ}$ that are not produced from the $3 \mathrm{ML}$ sample. If we refer to Fig. 9 we can see that these blocking dips are produced by holmium atoms in the upper region of the structure. We would not expect to see these features in a thinner silicide layer. In the hexagonal structure the dominant blocking dip is at a scattering angle of approximately $95^{\circ}$ (see fig. 


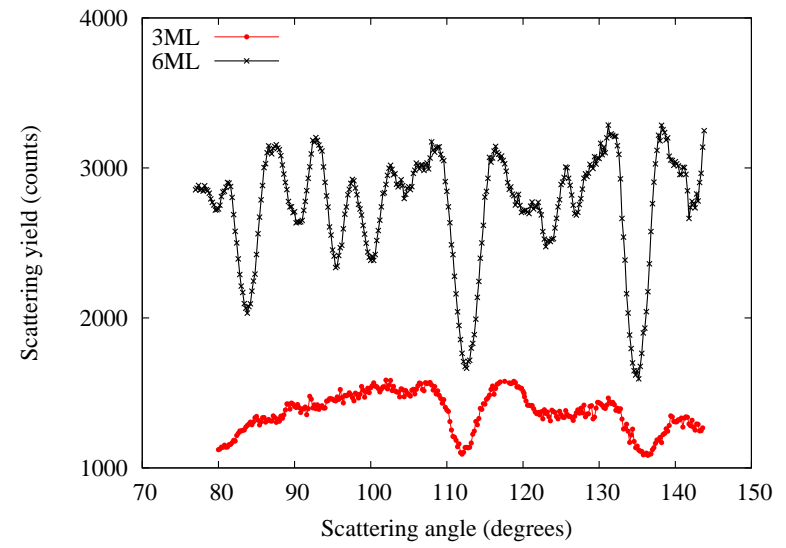

FIG. 11: Original in colour. A comparison of the $6 \mathrm{ML}$ coverage experimental data with data recorded after a sample was grown under identical growth conditions using a lower holmium coverage for the $[\overline{1} \overline{1} 0] /[1 \overline{1} 0]$ geometry. The vertical scales have been artificially offset to aid the eye.

4(a)d. The simulations suggest that this feature should be present in hexagonal silicides that contain as few as 3 layers of holmium. We can see no evidence for any blocking dips in this region in the experiment with $3 \mathrm{ML}$ coverage and we must conclude that even at this coverage the structure is tetragonal.

\section{SUMMARY AND CONCLUSION}

Holmium silicide islands have been grown on the $\mathrm{Si}(100)$ surface and characterised using MEIS. Two structures have been fitted to the experimental data and this process clearly shows that under our growth conditions it is the tetragonal phase of the silicide that is formed and not the hexagonal phase and that these two structures do not coexist on the surface (the orthorhombic phase is too similar to the tetragonal phase for us to be able to determine if some of the orthorhombic nature of bulk HoSi is present in this surface tetragonal phase). A further experiment using a lower holmium coverage of 3 ML has also been shown to have the tetragonal structure which confirms that the hexagonal phase is not the phase adopted at low coverages in this system.

Other authors have reported growth of the hexagonal phase under similar growth conditions for some of the other RE silicides. It is interesting to speculate as to why similar experiments have reached different structural conclusions. It would appear that the structure that is formed is very sensitive to the growth conditions. Island morphology is very sensitively dependent upon the particular RE metal deposited and the annealing temperature used. In the paper by $\mathrm{He}$ et al. 22] they report a deposition rate of $0.5 \mathrm{ML}$ per minute during the formation of $\mathrm{DySi}_{2}$ whereas Ye et al. [27] report 0.3 ML per minute. It could be that the mobility of the RE metal and/or the lattice mismatch anisotropy of the particular silicide in question are important enough factors during the very early stages of silicide formation to dictate the final structure.

Using a simple $1 \times 1 \times 2$ tetragonal unit cell the structure fitting suggests a $c$-axis value that is too small to be physically reasonable when compared to the bulk structure. The blocking curves from a large 2500 atom $10 \times 10 \times 2$ nanoisland with lateral strain relaxation as a function of the distance from the interface have been simulated. The major blocking dips produced by this structure are a better fit to the experimental data than those produced by a non-strained periodic structure. The lesser blocking dips produced by silicon atoms in the simulations do not show good agreement with experiment. This is to be expected because the thermal vibrations and the positions of these atoms have not been optimised in the structure fit. The results of the comparison of MEIS data with simulations clearly show that a physically reasonable $c$-axis value for the holmium silicide can only be obtained if the islands are strained to fit the $\mathrm{Si}(100)$ at the interface and the strain allowed to relax towards the top of the islands.

A full structural fit for this system would require an optimisation of the experiment-theory match with respect to all of the lateral and vertical spacings in the unit cell and their variation with distance from the interface, taking into account lateral strain relief, vertical relaxation and the particular vibrations of each individual atom. This task is not currently tractable in terms of computational resources and the available computer codes.

\section{Acknowledgments}

The authors would like to acknowledge the Engineering and Physical Science Research Council for funding this research. Paul Quinn and the FOM Institute are thanked for supplying the XVegas and Vegas simulation codes. The assistance and technical support of Kevin Connell and Mark Pendleton are also much appreciated.
[1] C. Preinesberger, S. Vandré, T. Kalka, and M. DähnePrietsch, J. Phys. D: Appl. Phys. 31, L43 (1998).

[2] A. Kirakosian, J. L. McChesney, R. Bennewitz, J. N. Crain, J.-L. Lin, and F. J. Himpsel, Surf. Sci. 498, L109
(2002).

[3] J. L. McChesney, A. Kirakosian, R. Bennewitz, J. N. Crain, J. L. Lin, and F. J. Himpsel, Nanotechnology 13, 545 (2002). 
[4] Y. Chen, D. A. A. Ohlberg, and R. S. Williams, J. Appl. Phys. 91, 3213 (2002).

[5] D. Lee and S. Kim, Appl. Phys. Lett. 82, 2619 (2003).

[6] B. Z. Liu and J. Nogami, Nanotechnology 14, 873 (2003).

[7] Y. Chen, D. A. A. Ohlberg, G. Medeiros-Ribeiro, and R. S. Williams, J. Crystal Growth 251, 657 (2003).

[8] J. Nogami, B. Z. Liu, M. V. Katkov, C. Ohbuchi, and N. O. Birge, Phys. Rev. B 63, 233305 (2001).

[9] B. Liu and J. Nogami, Surf. Sci. 488, 399 (2001).

[10] B. Z. Liu and J. Nogami, J. Appl. Phys. 93, 593 (2003).

[11] Y. Chen, D. A. A. Ohlberg, G. Medeiros-Ribeiro, Y. A. Chang, and R. S. Williams, Appl. Phys. Lett. 76, 4004 (2000).

[12] G. Chen, J. Wan, J. Yang, X. Ding, L. Ye, and X. Wang, Surf. Sci 513, 203 (2002).

[13] Q. Cai and W. Zhou, J. Phys.: Condens. Matter 16, 6835 (2004).

[14] C. Ohbuchi and J. Nogami, Phys. Rev. B 66, 165323 (2002).

[15] M. Katkov and J. Nogami, Bull. Am. Phys. Soc. 47, 283 (2002).

[16] N. Frangis, G. V. Tendeloo, J. V. Landuyt, G. Kaltsas, A. Travlos, and A. G. Nassiopoulos, Phys. Stat. Sol. (a) 158, 107 (1996).

[17] N. Frangis, J. V. Landuyt, G. Kaltsas, A. Travlos, and A. G. Nassiopoulos, J. Crystal Growth 172, 175 (1997).

[18] J. C. Chen, G. H. Shen, and L. J. Chen, J. Appl. Phys. 84, 6083 (1998).

[19] G. Chen, X. Ding, Z. Li, and X. Wang, J. Phys.: Condens. Matter 14, 10075 (2002).

[20] K. S. Chi, W. C. Tsai, and L. J. Chen, J. Appl. Phys. 93, 153 (2003).

[21] M. Kuzmin, P. Laukkanen, R. E. Perala, R. L. Vaara, and I. J. Varyrynen, Appl. Surf. Sci. 222, 394 (2004).

[22] Z. He, D. J. Smith, and P. A. Bennett, Phys. Rev. B 70, 241402(R) (2004).

[23] B. C. Harrison and J. J. Boland, Surf. Sci. 694, 93 (2005).

[24] G. Pető, G. Molnár, Z. E. Horváth, C. S. Daróczi, E. Zsoldos, and J. Gyulai, Surf. Sci. 578, 142 (2005).

[25] W. C. Tsai, H. C. Hsu, H. F. Hsu, and L. J. Chen, Appl. Surf. Sci. 244, 115 (2005).

[26] L. Pasquali and S. Nannarone, Nucl. Instrum. Methods Phys. Res. B 230, 340 (2005).

[27] G. Ye, J. Nogami, and M. A. Crimp, Thin Solid Films 497, 48 (2006).

[28] G. Ye, M. A. Crimp, and J. Nogami, Mater. Res. Soc. Symp. Proc. 901, Ra13 (2006).

[29] K. Maex and M. V. Rossum, eds., Properties of Metal Silicides (Institution of Engineering and Technology, 1995).

[30] C. Bonet, I. M. Scott, D. J. Spence, T. J. Wood, T. C. Q. Noakes, P. Bailey, and S. P. Tear, Phys. Rev. B 72, 165407 (2005).

[31] T. J. Wood, C. Bonet, T. C. Q. Noakes, P. Bailey, and S. P. Tear, Phys. Rev. B 73, 235405. (2006).

[32] D. J. Spence, S. P. Tear, T. C. Q. Noakes, and P. Bailey, Phys. Rev. B 61, 5707 (2000).

[33] D. J. Spence, T. C. Q. Noakes, P. Bailey, and S. P. Tear, Surf. Sci. 512, 61 (2002).

[34] D. J. Spence, T. C. Q. Noakes, P. Bailey, and S. P. Tear, Phys. Rev. B 62, 5016 (2000).

[35] T. J. Wood, C. Bonet, T. C. Q. Noakes, P. Bailey, and S. P. Tear, Surf. Sci. 598, 120 (2005).

[36] J. W. M. Frenken, J. F. van der Veen, and R. M. Tromp, Nucl. Instrum. Methods Phys. Res. B 17, 334 (1986). 